\title{
Delivery of progenitor cells with injectable shear-thinning hydrogel maintains geometry and normalizes strain to stabilize cardiac function after ischemia
}

\author{
Ann C. Gaffey, MD, MTR, ${ }^{a}$ Minna H. Chen, BChE, ${ }^{\mathrm{b}}$ Alen Trubelja, BS, ${ }^{\mathrm{a}}$ Chantel M. Venkataraman, BA,

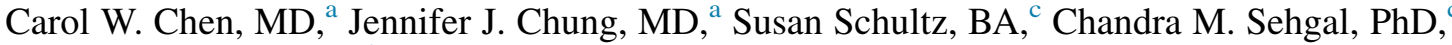 \\ Jason A. Burdick, PhD, ${ }^{b}$ and Pavan Atluri, $\mathrm{MD}^{\mathrm{a}}$
}

\section{ABSTRACT}

Objectives: The ventricle undergoes adverse remodeling after myocardial infarction, resulting in abnormal biomechanics and decreased function. We hypothesize that tissue-engineered therapy could minimize postischemic remodeling through mechanical stress reduction and retention of tensile myocardial properties due to improved endothelial progenitor cell retention and intrinsic biomechanical properties of the hyaluronic acid shear-thinning gel.

Methods: Endothelial progenitor cells were harvested from adult Wistar rats and resuspended in shear-thinning gel. The constructs were injected at the border zone of ischemic rat myocardium in an acute model of myocardial infarction. Myocardial remodeling, tensile properties, and hemodynamic function were analyzed: control (phosphate-buffered saline), endothelial progenitor cells, shear-thinning gel, and shear-thinning gel + endothelial progenitor cells. Novel high-resolution, high-sensitivity ultrasound with speckle tracking allowed for global strain analysis. Uniaxial testing assessed tensile biomechanical properties.

Results: Shear-thinning gel + endothelial progenitor cell injection significantly increased engraftment and retention of the endothelial progenitor cells within the myocardium compared with endothelial progenitor cells alone. With the use of strain echocardiography, a significant improvement in left ventricular ejection fraction was noted in the shear-thinning gel + endothelial progenitor cell cohort compared with control $(69.5 \% \pm 10.8 \%$ vs $40.1 \% \pm 4.6 \%, P=.04)$. A significant normalization of myocardial longitudinal displacement with subsequent stabilization of myocardial velocity with shear-thinning gel + endothelial progenitor cell therapy compared with control was also evident $(0.84+0.3 \mathrm{~cm} / \mathrm{s}$ vs $0.11 \pm 0.01 \mathrm{~cm} / \mathrm{s}, P=.03)$. A significantly positive and higher myocardial strain was observed in shear-thinning gel + endothelial progenitor cell $(4.5 \% \pm 0.45 \%)$ compared with shear-thinning gel $(3.7 \% \pm 0.24 \%)$, endothelial progenitor cell $(3.5 \% \pm 0.97 \%)$, and control $(8.6 \% \pm 0.3 \%, P=.05)$. A resultant reduction in dynamic stiffness was noted in the shear-thinning gel + endothelial progenitor cell cohort.

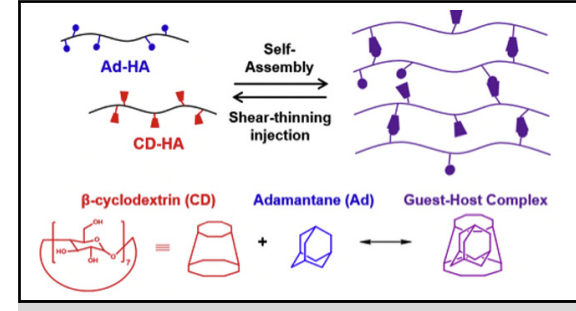

Shear-thinning hydrogel with self-healing.

\section{Central Message}

Injectable shear-thinning HA hydrogel demonstrates stabilization of border zone myocardium with a reduction in adverse myocardial remodeling and preservation of myocardial biomechanics.

\section{Perspective}

The tissue-engineered STG used in this study may improve therapies by allowing investigators to stabilize the at-risk border zone myocardium, to maintain ventricular geometry, to enhance the delivery of cellular therapy with significant cellular retention at targeted sites, and to improve ventricular hemodynamics.

See Commentary page 1491.

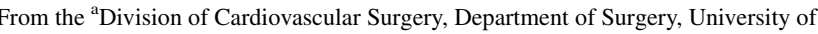
Pennsylvania, Philadelphia, $\mathrm{Pa}$; and Departments of ${ }^{\mathrm{b}}$ Bioengineering and ${ }^{\mathrm{c}}$ Radiology, University of Pennsylvania, Philadelphia, Pa.

Funding: The National Institutes of Health (R01 HL107938, R01 HL111090) and American Heart Association through a predoctoral fellowship (to C.B.R.) and established investigator award (to J.A.B.). The American Association for Thoracic Surgery, David C. Sabiston Research Scholarship (to P.A.), and the American Heart Association, Scientist Development Grant 13SDG17230005 (to P.A.).

Read at the 97th Annual Meeting of The American Association for Thoracic Surgery, Boston, Massachusetts, April 29-May 3, 2017.
}

Received for publication July 14, 2017; revisions received July 26, 2018; accepted for publication July 27, 2018; available ahead of print Dec 20, 2018.

Address for reprints: Pavan Atluri, MD, Division of Cardiovascular Surgery, Department of Surgery, University of Pennsylvania, Silverstein 6, 3400 Spruce St, Philadelphia, PA 19104 (E-mail: Pavan.atluri@uphs.upenn.edu).

$0022-5223 / \$ 36.00$

Copyright $\left({ }^{\circ} 2018\right.$ by The American Association for Thoracic Surgery https://doi.org/10.1016/j.jtcvs.2018.07.117 


$$
\begin{array}{ll}
\text { Abbreviations and Acronyms } \\
\text { CD } & =\beta \text {-cyclodextrin } \\
\text { EPC } & =\text { endothelial progenitor cell } \\
\text { HA } & =\text { hyaluronic acid } \\
\text { hpf } & =\text { high-power field } \\
\text { LAD } & =\text { left anterior descending } \\
\text { LV } & =\text { left ventricular } \\
\text { MI } & =\text { myocardial infarction } \\
\text { PBS } & =\text { phosphate-buffered saline } \\
\text { STG } & =\text { shear-thinning gel } \\
\text { STG }+ \text { EPC } & =\text { shear-thinning gel endothelial } \\
& \text { progenitor cell construct } \\
\text { TBA } & =\text { tetrabutylammonium hydroxide }
\end{array}
$$

Conclusions: This novel injectable shear-thinning hyaluronic acid hydrogel demonstrates stabilization of border zone myocardium with reduction in adverse myocardial remodeling and preservation of myocardial biomechanics. The cellular construct provides a normalization of strain measurements and reduces left ventricular dilatation, thus resulting in improvement of left ventricular function. ( $\mathrm{J}$ Thorac Cardiovasc Surg 2019;157:1479-90)

Approximately 600,000 Americans experience a new myocardial infarction (MI) each year, and 300,000 Americans experience a recurrent infarction. ${ }^{1}$ Advances in cardiovascular care have allowed the majority of patients to survive their initial MI. However, the treatment strategies for patients who later develop ventricular dysfunction are still lacking. Approximately $70 \%$ of postischemic heart failure cases are attributed to left ventricular (LV) remodeling that is manifested by progressive LV dilation and loss of global contractile function.

After an MI, infarct expansion initiates and mediates progressive adverse LV remodeling. Remodeling results from increased mechanical loads due to intracavity pressure and force generated from noninfarcted myocardium of the ventricle as a result of infarcted noncontractile myocardium during the cardiac cycle. Such remodeling follows the principle of Laplace's law, as the left ventricle dilates and the LV wall thins, wall stress and myocardial oxygen consumption increase, resulting in periodic subendocardial ischemia and continued myocardial damage. This deleterious effect causes abnormal myocardial loading and induces stretching of the infarct with increased mechanical stress at the border zone region. ${ }^{2}$ As remodeling continues, additional perfused myocardium becomes hypocontractile. Adverse remodeling involves progressive expansion of the border zone, leading to further diminution of pump function, loss of global contractile function, and ultimately heart failure. ${ }^{3}$ Over time, these abnormal stress distributions lead to acquired biological changes within the myocardium and a dysfunctional cardiac cycle. ${ }^{4}$

LV augmentation with the intramyocardial injection of an alginate-based polymer has been shown to result in increased wall thickness and preservation of LV geometry. ${ }^{5}$ To reduce the negative sequela of ischemic injury and improve global myocardial function, we have tested the use of endothelial progenitor cells (EPCs) encapsulated within a shear-thinning gel (STG) to treat ischemic myocardium. The premise of EPC effectiveness is based on postnatal angiogenesis to revascularize the ischemic myocardium. Our laboratory and others have demonstrated that EPCs are easily cultured in vitro and have the ability to engraft, improve cardiac function, and attenuate ventricular remodeling when delivered after infarction. ${ }^{6-10}$ Unfortunately, clinical translation of this therapy has been severely limited. One of the major challenges of the therapeutic implementation of EPCs has been the optimization of cell delivery and engraftment to minimize cell dispersal. After direct cell injection into the myocardium, less than $1 \%$ of the cells are retained as shown by tracking studies. ${ }^{11-15}$ Through the use of a biomaterial to provide a surrogate extracellular matrix to localize the EPCs at the delivery site, we have shown the ability to enhance cell delivery in a hospitable environment. ${ }^{6}$ Our STG is based on hyaluronic acid (HA), a liner polysaccharide found in native cardiac extracellular matrix that plays a role in cardiac embryogenesis, scar reduction, cell migration, and angiogenesis. ${ }^{16}$

We aim to investigate this treatment further by evaluating the therapeutic implications on regional biomechanics of the infarct region. Most notably, strain, which is calculated by change in length divided by the original lengths in the circumferential and radial directions, will be calculated using a speckle-tracking algorithm (EchoPAC, GE Healthcare, Chicago, Ill). An in-depth analysis is expected to demonstrate a normalization in regional strain in the periinfarct myocardium after treatment with the shearthinning gel endothelial progenitor cell construct (STG + EPC). These experiments are motivated by the understanding that global and border zone mechanical dysfunction are key steps in the progression to heart failure. We hypothesize that the construct of the STG with incorporated EPCs will allow for higher cellular retention rates within the ischemic myocardium as assessed through near-infrared imaging. Because of the enhanced cell retention, we further hypothesize that our therapy will limit postinfarction adverse ventricular remodeling resulting in preservation of cardiac function. 


\section{MATERIALS AND METHODS} Animal Care and Biosafety

Male adult Wistar rats (250-300 g) were obtained from Charles River Laboratories (Boston, Mass). Food and water were provided ad libitum. This investigation adheres to the Guide for the Care and Use of Laboratory Animals published by the US National Institutes of Health (8th ed, revised 2011). This study conforms to institutional ethical review and has been approved by the University of Pennsylvania Institutional Animal Care and Use Committee (Protocol 803394).

\section{Isolation, Culture, and Characterization of Bone Marrow Endothelial Progenitor Cells}

Bone marrow mononuclear cells were isolated from the long bones of syngeneic adult, male Wistar rats by density-gradient centrifugation (Histopaque 1083; Sigma-Aldrich, St Louis, Mo). After isolation, the cells were seeded on vitronectin-coated plates. The cells were cultured in endothelial basal medium supplemented with human epidermal growth factor, fetal bovine serum, vascular endothelial growth factor, human fibroblast growth factor-B, R3-insulin-like growth factor, ascorbic acid, gentamicin, and amphotericin-B for 6 days (Lonza, Basel, Switzerland). EPC phenotype $\left(\right.$ DiLDL $^{+}$VEGFR $^{+} \mathrm{CD}^{+} 4^{+}$) was previously confirmed by our group using flow cytometry and immunocytochemistry. 8,17

\section{Endothelial Progenitor Cell Shear-Thinning Hydrogel Construct Creation}

To form the shear-thinning HA gel, sodium HA $(90 \mathrm{kDa})$ was purchased from Lifecore (Chaska, Minn). All other chemicals were purchased from Sigma-Aldrich. The sodium salt of HA was dissolved in deionized water at 2 wt \%, exchanged against Dowex-100 resin, neutralized by tetrabutylammonium hydroxide (TBA), frozen, and lyophilized to form HA-TBA. Adamantane-modified HA was prepared by coupling 1-adamantane acetic acid to HA-TBA via esterification, as previously described. ${ }^{16} \beta$-cyclodextrin $(\mathrm{CD})$ was first reacted to add a hexane diamine linker to form $\beta$-CDhexane diamine, which was coupled via amidation to HA-TBA to form $\beta$-cyclodextrin modified HA (CD-HA) (Figure 1). Percent modification of the HA backbone by adamantane $(26 \%)$ or $\beta$-cyclodextrin $(21 \%)$ was confirmed by nuclear magnetic resonance. Hydrogels of $4 \mathrm{wt} \%$ total polymer concentration were then subsequently prepared from stock solutions of Adamantane-modified HA and CD-HA in phosphate-buffered saline (PBS). To ensure guest-host assembly mechanism, the 2 component solutions were combined and mixed manually by stirring to ensure a homogenous structure, which was then briefly vortexed to remove entrapped air. The shear-thinning and viscoelastic behavior of this gel were confirmed by oscillatory rheology. Viscosity decreased with increasing shear rate
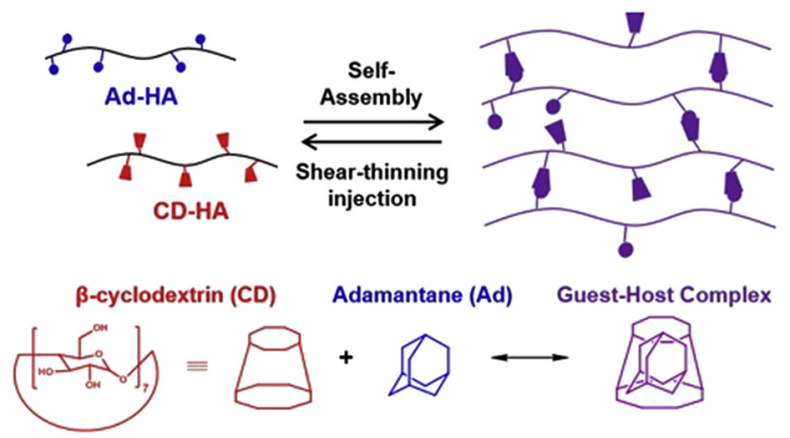

FIGURE 1. Overview of hydrogel composition. Schematic of STG formation, using guest-host complex of CD (host) and adamantane (guest) for initial self-assembly, as well as subsequent injection and self-healing in situ. $A d$, Adamantine; $C D, \beta$-cyclodextrin; $H A$, hyaluronic acid. and stress, displaying classical shear-thinning behavior. With increasing strain $\%$, gels yielded and the storage modulus $\left(\mathrm{G}^{\prime}\right)$ dropped below the loss modulus $\left(\mathrm{G}^{\prime \prime}\right)$ to permit flow as shown in prior work by our collaborators. ${ }^{16}$ For incorporation of the EPCs, the cells were trypsinized and counted before being mixed into the gel to ensure a cell density of 700,000 cells per $100 \mu \mathrm{L}$ of STG. This cellular density has demonstrated improvements in angiogenesis as shown by our group in a previous study. 6,18

\section{Induction of Myocardial Infarction and Intramyocardial Endothelial Progenitor Cell Delivery}

Animal studies were performed in accordance with the University of Pennsylvania Animal Ethics Committee. A total of 48 rats $(n=12)$ were initially allocated to each arm. After left anterior descending (LAD) ligation, there is approximately a $15 \%$ incidence of lethal arrhythmia and those animals were excluded from the study. Male Wistar rats $(\sim 300 \mathrm{~g})$ were anesthetized with $2.5 \%$ isoflurane in an induction chamber (VetEquip, Pleasanton, Calif), endotracheally intubated, and mechanically ventilated (Harvard Apparatus, Holliston, Mass) with 1\% isoflurane. A left lateral thoracotomy in the fourth intercostal space was performed. The proximal LAD was identified approximately $2 \mathrm{~mm}$ below the left atrial appendage, and a 7-0 Prolene suture (Ethicon, Somerville, NJ) was used to ligate the artery. Regional LV ischemia was confirmed by immediate discoloration of the myocardium and change in the cardiac rhythm. This technique creates a consistent and reproducible anterolateral infarction of 35\% to $40 \%$ of the left ventricle. ${ }^{8,19-21}$ After ligation, the exposed heart was monitored for 5 minutes for stability and return to sinus rhythm.

After LAD ligation, animals were randomized to 1 of 4 groups: control (LAD ligation with injection of PBS, $n=10$ ), intramyocardial injection of EPCs alone (700,000 cells in $100 \mu \mathrm{L}$ PBS, $\mathrm{n}=9$ ), injection of blank STG $(100 \mu \mathrm{L}, \mathrm{n}=9)$, and treatment with STG $+\mathrm{EPC}(\mathrm{n}=11)$. A total of $100 \mu \mathrm{L}$ was delivered to the myocardium with a straight 27 -gauge needle into 5 sites along the anterior and posterior LV wall at the border between the ischemic myocardium and the viable muscle. After ligation of the LAD, a clear color change exists in the distribution of the infarct, and at approximately 0.5 to $1.0 \mathrm{~mm}$ from the discolored myocardium the injections of the border zone myocardium are performed. The chest was then closed in 3 layers, and the animals were allowed to recover and be extubated. Four weeks after MI, hemodynamic analysis was conducted and the animals were killed for histologic analysis.

\section{Quantification of Myocardial Angiogenesis}

At the 4-week time point after LAD ligation and treatment, the hearts were explanted. Sections were washed 3 times in PBS, fixed in $4 \%$ paraformaldehyde for 10 minutes at room temperature, and blocked in $10 \%$ fetal bovine serum (Gibco, Gaithersburg, Md) for 1 hour at $37^{\circ} \mathrm{C}$. Primary antibodies were diluted 1:150 in PBS and incubated for 2 hours at $37^{\circ} \mathrm{C}$. Secondary antibodies were diluted in PBS and incubated for 2 hours at $37^{\circ} \mathrm{C}$. Primary antibodies included sheep anti-von Willebrand factor conjugated to fluorescein isothiocyanate (Abcam [Cambridge, Mass], ab8822) and rabbit anti-smooth muscle actin (Abcam, ab5694). Donkey anti-rabbit conjugated to Alexa Fluor 594 (Abcam, ab150068) was used as a secondary antibody (1:200). Nuclei were stained with DAPI (Vector Laboratories, Burlingame, Calif). Vasculature was quantified using ImageJ (National Institutes of Health, Bethesda, Md). Measurements were made 1 high-power field (hpf) from the infarct at $20 \times$ magnification.

\section{Analysis of Global Ventricular Function}

Before explanting of the heart, transthoracic echocardiography was performed to assess myocardial function (VSI Vevo 2100, VisualSonics, Toronto, Ontario, Canada). Ventricular measurements were performed 
according to the American Society for Echocardiography leading-edge method. Additionally, a 2F pressure-volume catheter (Millar Instruments, Houston, Tex) was inserted into the left ventricle in a retrograde fashion via the right common carotid artery for analysis of ventricular function. In addition to steady-state hemodynamic parameters, contractility was determined from pressure-volume relationships obtained by measuring the ventricular response to preload reduction via occlusion of the inferior vena cava. All measurements were performed by 2 independent investigators blinded to animal treatment.

\section{Analysis of Myocardial Biomechanics}

Echocardiography images were obtained in bidimensional (2-dimensional), M-mode, and strain (speckle-tracking) examinations. Rats were anesthetized using $2.5 \%$ isoflurane for induction and were placed in a supine position. Examinations were performed by the same blinded echocardiographer following the recommendations of the American Society of Echocardiography.

Echocardiographic speckle-tracking-based strain measures of myocardial deformation were obtained from 2-dimensional gray-scale echocardiography images acquired from the parasternal long view (VSI Vevo 2100, VisualSonics). Strain, strain rate, velocity, and displacement of myocardial segments were quantified in the longitudinal axis. All images were acquired at a frame rate of 220 frames per second and $3 \mathrm{~cm}$ depth.

Strain analyses were conducted using the speckle-tracking algorithm provided by VisualSonics (VevoStrain, VisualSonics). In brief, 3 consecutive cardiac cycles in B-mode loops were selected from digitally acquired echocardiography images based on adequate visualization of the endocardial border and absence of image artifacts. Semiautomated tracing of the endocardial and epicardial borders were performed and verified over all 3 cardiac cycles and corrected as needed to achieve quality tracking throughout each cine loop. Tracked images were processed in a frameby-frame manner for strain measurements. Strain represents a measure that evaluates the degree of deformation of the analyzed segment in relation to its initial dimensions, and it is expressed as percentage. The strain equation is as follows:

$$
\varepsilon=L-L_{0} / L_{0}
$$

Where $\mathrm{L}$ is the length of the object after deformation, and $\mathrm{L}_{0}$ is the basal length of the object. By convention, depending on the direction, a lengthening or thickening deformation is given a positive value, whereas a shortening or thinning deformation is given a negative one. Each long-axis view of the left ventricle myocardium was divided into 6 standard anatomic segments for regional speckle-tracking-based strain analysis throughout the cardiac cycle. To obtain global values, velocity, displacement, and strain rate measurements were averaged across all 6 segments and the border zone. The border zone was defined as anterior apex, posterior apex, and posterior mid-ventricle.

\section{Analysis of Uniaxial Biomechanical Testing}

Specimens were placed in a $37^{\circ} \mathrm{C}$ temperature-regulated PBS bath and mounted to an Instron 5548 Material Testing System with a $5 \mathrm{~N}$, 6-axis load cell. Specimens were first preloaded at $0.05 \mathrm{~N}$ for gauge length recording. A total of 20 cycles of preconditioning were performed $(0.05-0.1 \mathrm{~N}$ at a rate of $0.1 \mathrm{~mm} / \mathrm{sec}$ ). After a 60 -second hold at $0.05 \mathrm{~N}, 3$ increments of stressrelaxation were performed with each increment displaced rapidly by an additional $0.5 \mathrm{~mm}$ at the rate of $0.5 \mathrm{~mm} / \mathrm{sec}$. There was a 300 -second hold period between each increment, where the extension was held constant. The dynamic loading protocol was performed at $5 \%$ and $10 \%$ strain, and included stress relaxation and sinusoidal frequency sweeps. Each frequency sweep included 10-cycle oscillations (amplitude: 0.125\% strain, frequency: 0.1 and $1.0 \mathrm{~Hz}$ ). After a return to gauge length (down $1.5 \mathrm{~mm}$ at $-0.5 \mathrm{~mm} / \mathrm{sec}$ ) and a 60 -second hold, a ramp to failure at a rate of $0.1 \mathrm{~mm} / \mathrm{sec}$ was performed. ${ }^{22-24}$ Load data were captured continuously in Bluehill 2 (Instron, Norwood, Mass), and LabVIEW (National Instruments, Austin, Tex) was used to acquire optical data at a sampling rate of 1 frame/sec using a custom-built algorithm. Dynamic stiffness was calculated from the force-displacement data.

\section{Analysis of Bioluminescent Tracking of Delivered Endothelial Progenitor Cells}

CellVue NIR815 (Li-Cor, Lincoln, Neb) is an aliphatic near-infrared dye that stably incorporates into the cell membrane lipid bilayer without disrupting cell viability or function. EPCs were labeled immediately before injection using an iso-osmotic aqueous diluent with $2 \times 10^{-6} \mathrm{~mol} / \mathrm{L}$ CellVue NIR815 dye to induce noncovalent intercalation of the dye in the cell membrane. Trypan blue viability test of cells postlabeling confirmed the nontoxic properties of NIR815. Labeled EPCs were encapsulated in the STG and delivered to ischemic myocardium. NIR815-tagged EPCs were visualized using the LI-COR Pearl Small Animal Imaging System (Lincoln, Neb).

\section{Statistical Analysis}

Treatment across groups was randomly generated with animals coded by random identifiers. The sample size of $\mathrm{n}=11$ will allow the width of a $95 \%$ confidence interval of no more than 1 standard deviation assuming normally distributed data. Investigators were blinded to treatment group during data acquisition and analysis. All values are expressed as the mean \pm standard error of the mean. Statistical significance was evaluated using unpaired 2-sample $t$ tests with unequal variance. Normality of the data was tested using the Shapiro-Wilk test. Comparison of data across all 4 experimental groups was performed by 2 -way analysis of variance. Scatter plots show mean values with standard error of the mean error bars. All analyses were performed using Stata 13.1 (StataCorp LP, College Station, Tex).

\section{RESULTS}

\section{Shear-Thinning Gel and Endothelial Progenitor Cell Construct Enhances Myocardial Angiogenesis}

In our previous work with STG-encapsulated EPCs, we showed the gel vehicle potentiated the effect of EPCs by minimizing cell dispersion. ${ }^{6}$ Quantification of myocardial angiogenesis demonstrates a statistically significant increase in vascular density within the ischemic myocardium after treatment with the STG-EPC construct $(15.3 \pm 5.8$ vessels/hpf) compared with the control $(3.8 \pm 2.4$ vessels/ hpf, $P<.0001)$. Interestingly, the significant improvement in angiogenesis was not evident after isolated cell injection $(5.0 \pm 2.1$ vessels/hpf) compared with control $(3.8 \pm 2.4$ vessels/hpf, $P=.30$ ). This suggests that enhanced cell retention may contribute to significantly enhanced angiogenesis within the ischemic myocardium.

\section{Improvement in Global Ventricular Function}

Hemodynamic analysis demonstrated a statistically significant improvement in LV ejection fraction, maximum pressure, $\mathrm{dP} / \mathrm{dt}$ max, end-diastolic volume, and contractility (Table 1). The improvement in ejection fraction within the STG + EPC group $(69.5 \% \pm 10.8 \%)$ was significantly greater than the control $(40.1 \% \pm 4.6 \%)$, EPC $(42.3 \% \pm 8.7 \%)$, and STG groups $(54.6 \% \pm 9.2 \%$, $P=.04)$. The maximum pressure generated was 
TABLE 1. Left ventricular function 4 weeks after left anterior descending coronary artery ligation and treatment using echocardiography and Millar catheter

\begin{tabular}{|c|c|c|c|c|c|}
\hline & Control $(n=10)$ & $\operatorname{EPC}(\mathbf{n}=9)$ & STG $(\mathbf{n}=9)$ & STG $+\operatorname{EPC}(n=11)$ & $P$ value \\
\hline LV ejection fraction $(\%)$ & $40.1 \pm 4.6$ & $42.3 \pm 8.7$ & $54.6 \pm 9.2$ & $69.5 \pm 10.8$ & .04 \\
\hline Maximum pressure $(\mathrm{mm} \mathrm{Hg})$ & $51.3 \pm 5.3$ & $76.1 \pm 5.8$ & $90.3 \pm 7.0$ & $99.4 \pm 9.1$ & .01 \\
\hline $\mathrm{dP} / \mathrm{dt} \max (\mathrm{mm} \mathrm{Hg} / \mathrm{sec})$ & $2102 \pm 76.3$ & $2249 \pm 80.9$ & $3250 \pm 362.1$ & $5741 \pm 296.2$ & .01 \\
\hline End-diastolic volume $(\mu \mathrm{L})$ & $217.6 \pm 80.4$ & $243.3 \pm 126.3$ & $114.9 \pm 58.6$ & $112.0 \pm 78.5$ & .05 \\
\hline Contractility $(\mu \mathrm{L} / \mathrm{mm} \mathrm{Hg})$ & $1.2 \pm 0.3$ & $1.6 \pm 0.4$ & $2.1 \pm 0.3$ & $3.1 \pm 0.1$ & .05 \\
\hline
\end{tabular}

$E P C$, Endothelial progenitor cell; $L V$, left ventricular; $S T G$, shear-thinning gel.

significantly greater in the STG + EPC group $(99.4 \pm 9.1 \mathrm{~mm} \mathrm{Hg})$ compared with the control $(51.3 \pm 5.3 \mathrm{~mm} \mathrm{Hg})$, EPC $(76.1 \pm 5.8 \mathrm{~mm} \mathrm{Hg})$, and STG $(90.3 \pm 7.0 \mathrm{~mm} \mathrm{Hg}, P=.01)$ groups. As shown in Figure 2, there was improved contractility within the $\mathrm{STG}+$ EPC group $(3.1 \pm 0.1 \mu \mathrm{L} / \mathrm{mm} \mathrm{Hg})$ compared with the STG $(2.1 \pm 0.3 \mu \mathrm{L} / \mathrm{mm} \mathrm{Hg})$, EPC $(1.6 \pm 0.4 \mu \mathrm{L} / \mathrm{mm}$ $\mathrm{Hg})$, and control $(1.2 \pm 0.2 \mu \mathrm{L} / \mathrm{mm} \mathrm{Hg})$ groups. As indicated by the normalization of LV end-diastolic volume, there was preservation of ventricular geometry. The $\mathrm{STG}+\mathrm{EPC}$ cohort $(112.0 \pm 78.5 \mu \mathrm{L}, P=.05)$ displayed improved contractility and function. The LV end-diastolic volume was noted to be greater within the control $(217.6 \pm 80.4 \mu \mathrm{L})$, EPC $(243.3 \pm 126.3 \mu \mathrm{L})$, and STG groups $(114.9 \pm 58.6 \mu \mathrm{L})$ when compared with STG + EPC $(P=.05)$ as shown in Table 1.

\section{Retention of Myocardial Velocity and Reduction in Myocardial Strain}

For evaluation of regional myocardial strain and function, the LV was divided into 6 segments (anterior, anteroseptal, inferoseptal, inferior, inferolateral, and anterolateral). The strains, which are changes in length divided by the original lengths in the circumferential and radial direction, were calculated using a speckletracking algorithm. The sum of the areas of the anterior and anterolateral segments was defined as the infarct area. The sum of the areas of the anteroseptal and

\section{PBS}

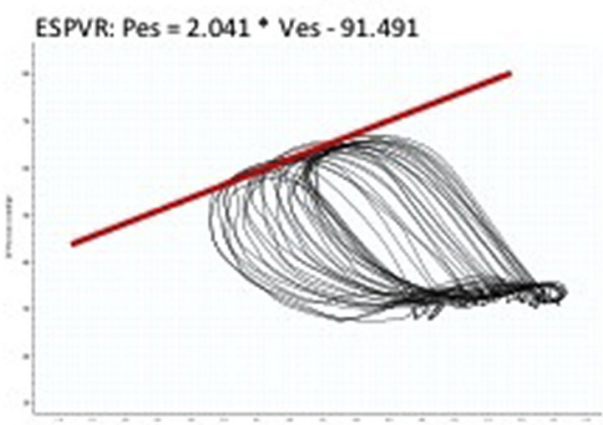

\section{STG}

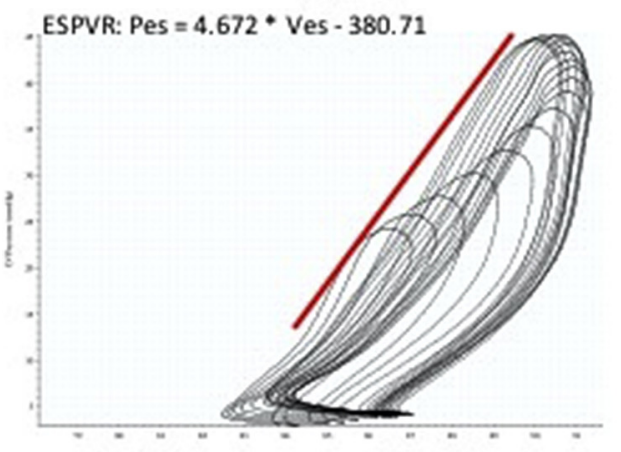

EPC

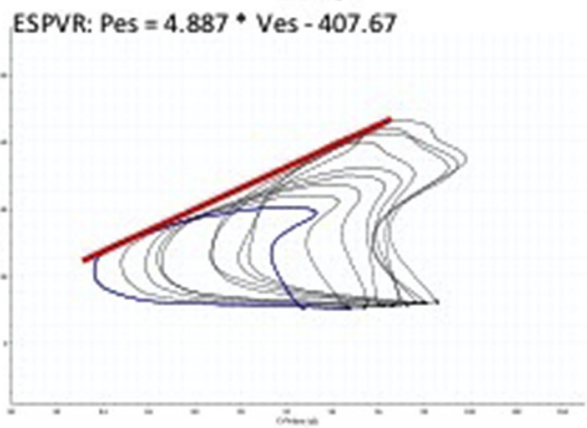

STG+EPC

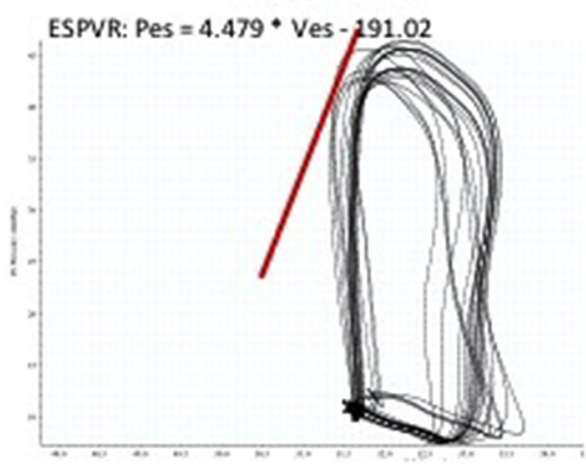

FIGURE 2. In vivo hemodynamic measurements after LAD ligation and treatment. Representative P-V loop during inferior vena cava occlusion per treatment group depicting a higher maximum pressure, a greater ejection fraction, and markedly better contractility in the STG + EPC group. $E S P V R$, end-systolic pressure-volume relationship; $E P C$, endothelial progenitor cell; $P B S$, phosphate-buffered saline; $S T G$, shear-thinning gel. 
inferolateral segments was described as the peri-infarct area, and the sum of the areas of the inferoseptal and inferior segments was the remote area. Within the graphic depiction, red represents hypocontractile myocardium, white is reduced contractility myocardium, and blue is dynamic/normal myocardium. As shown in Figure 3, within the PBS group there is global loss of normal ventricular geometry, suggesting an expansion of the dysfunctional myocardium after the infarct. With EPC injection alone, there was little improvement in the border zone velocity and greater dysfunctional myocardium supporting an expansion of the infarct as evident by the increase in red segments. A slight improvement after STG treatment was observed within the border zone as evident by the reduced contractile myocardium. Within the STG + EPC construct treatment area, there was normalization of border zone velocity within the epicardium and endocardium. The ventricular velocity remained high with small rate to peak contraction, indicating preservation of the ventricular geometry. Preservation of LV velocity with treatment was further supported by the normalization of border zone displacement of the epicardium to $0.55 \pm 0.2 \mathrm{~mm}$ in the STG + EPC group compared with control $(0.14 \pm 0.1 \mathrm{~mm})$, EPC $(0.38 \pm 0.2 \mathrm{~mm})$, and STG $(0.47 \pm 0.4 \mathrm{~mm}, P=.03)$ as shown in Table 2 .

The STG + EPC group tolerated a higher myocardial strain $(8.6 \% \pm 0.3 \%)$, compared with PBS $(3.7 \%+0.24 \%)$, EPC $(3.5 \% \pm 0.97 \%)$, and STG $(4.5 \% \pm 0.45 \%, P=.05)$. The higher strain percentage supports a lengthening and thickening deformation, and the lower value gives a shortening or thinning deformation. Likewise, a significantly higher myocardial strain rate was observed in STG + EPC $(31.3 \pm 7.21 / \mathrm{ms})$ compared with PBS $(12.3 \pm 0.81 / \mathrm{ms})$, EPC $(15.6 \pm 3.21 / \mathrm{ms})$, and STG $(30.8 \pm 8.11 / \mathrm{ms}, P=.03)$ as noted in Table 2 .
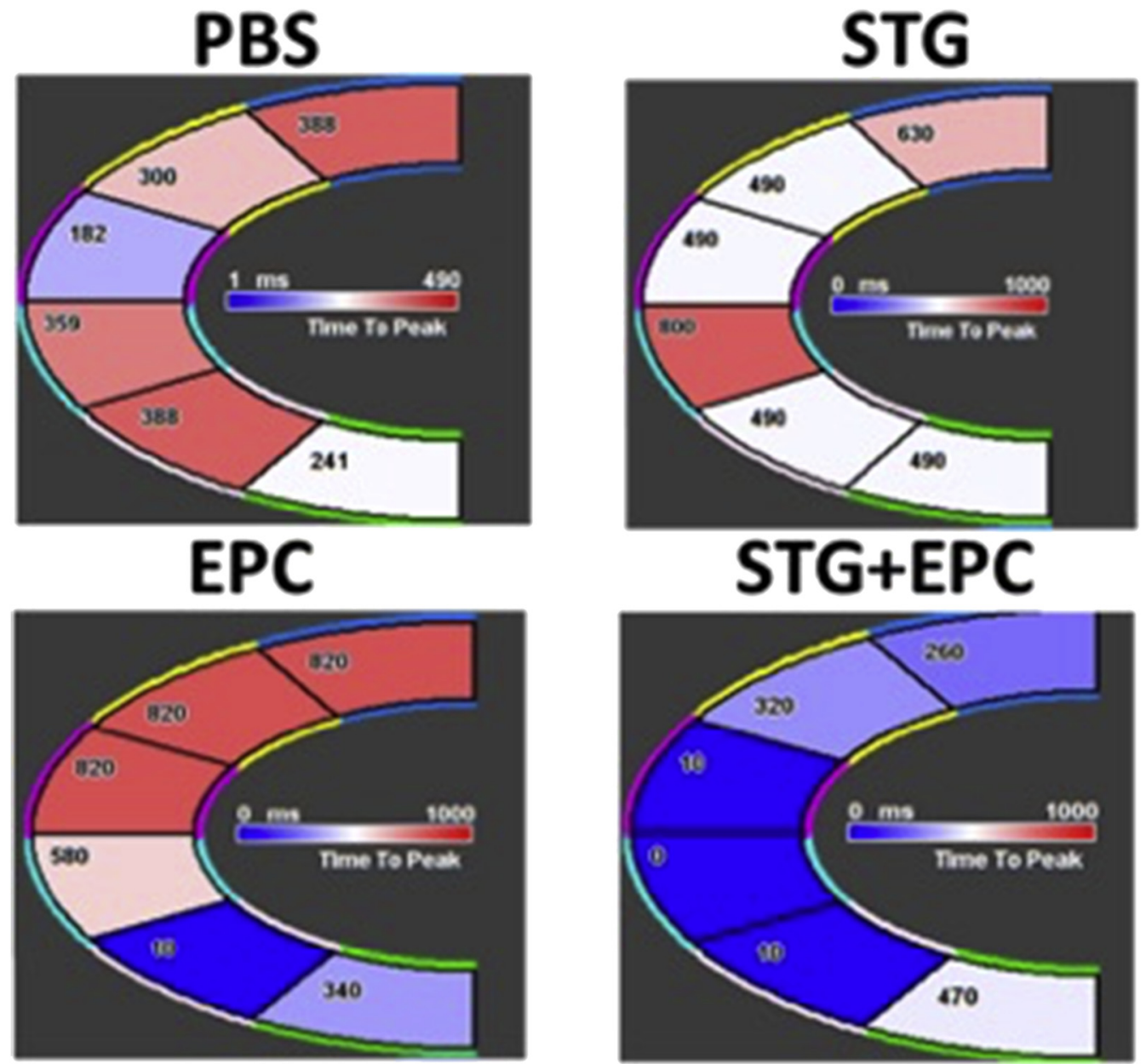

FIGURE 3. High-resolution ultrasound with speckle-tracking echocardiogram displaying the segmental velocities of the left ventricle after MI and randomization to treatment group (red = hypocontractile myocardium, white $=$ reduced contractility myocardium, and blue $=$ dynamic/normal myocardium). EPC, Endothelial progenitor cell; PBS, phosphate-buffered saline; $S T G$, shear-thinning gel. 
TABLE 2. Strain echocardiography examination

\begin{tabular}{|c|c|c|c|c|c|}
\hline Column 1 & Control $(\mathbf{n}=10)$ & $\mathbf{E P C}(\mathbf{n}=9)$ & STG $(\mathbf{n}=9)$ & STG $+\operatorname{EPC}(n=11)$ & $P$ value \\
\hline Fractional shortening $(\%)$ & $31.9 \pm 1.9$ & $37.8 \pm 3.5$ & $54.9 \pm 12.7$ & $56.3 \pm 7.8$ & .05 \\
\hline Peri-infarct regional myocardial strain $(\%)$ & $3.7 \pm 0.24$ & $.3 .5 \pm 0.97$ & $4.5 \pm 0.45$ & $8.6 \pm 0.3$ & .55 \\
\hline Peri-infarct regional myocardial strain rate $(1 / \mathrm{ms})$ & $12.3 \pm 0.8$ & $15.6 \pm 3.2$ & $30.8 \pm 8.1$ & $31.3 \pm 7.2$ & .05 \\
\hline Global displacement epicardium (mm) & $0.14 \pm 0.1$ & $0.38 \pm 0.2$ & $0.67 \pm 0.4$ & $0.66 \pm 0.2$ & .42 \\
\hline Border zone displacement epicardium (mm) & $0.20 \pm 0.1$ & $0.26 \pm 0.1$ & $0.46 \pm 0.4$ & $0.58 \pm 0.1$ & .05 \\
\hline Global endocardium velocity $(\mathrm{cm} / \mathrm{s})$ & $0.11 \pm 0.01$ & $0.36 \pm 0.1$ & $0.31 \pm 0.2$ & $0.84 \pm 0.3$ & .03 \\
\hline Border zone endocardium velocity $(\mathrm{cm} / \mathrm{s})$ & $0.19 \pm 0.03$ & $0.29 \pm 0.1$ & $0.66 \pm 0.8$ & $0.73 \pm 0.4$ & .05 \\
\hline Global epicardium velocity $(\mathrm{cm} / \mathrm{s})$ & $0.16 \pm 0.05$ & $0.47 \pm 0.2$ & $0.55 \pm 0.2$ & $0.84 \pm 0.4$ & .05 \\
\hline Border zone epicardium velocity $(\mathrm{cm} / \mathrm{s})$ & $0.30 \pm 0.3$ & $0.72 \pm 0.6$ & $0.78 \pm 0.5$ & $0.82 \pm 0.4$ & .06 \\
\hline
\end{tabular}

$E P C$, Endothelial progenitor cell; $S T G$, shear-thinning gel.

\section{Restoration of Myocardial Viscoelastic Properties}

A custom software package running in MATLAB (MathWorks, Natick, Mass) was used to analyze the load and optical data. Load data were divided by the cross-sectional area to determine stress, and strain was obtained by selecting 2 regions of interest for each strain line. The viscoelastic properties were quantified by analyzing the incremental stress-relaxation ramps. Specifically, we determined the ratio of the stress $(\mathrm{Pa})$ to the percentage strain (Figure 4). At $5 \%$ strain, a significant reduction in stress was observed at $0.1 \mathrm{~Hz}$ for the STG + EPC $(0.16 \pm 0.12 \mathrm{~Pa} / \%$ strain $)$ compared with PBS $(0.42 \pm 0.30 \mathrm{~Pa} / \%$ strain $)$, EPC $(0.34 \pm 0.31 \mathrm{~Pa} / \%$ strain $)$, and STG $(0.20 \pm 0.13 \mathrm{~Pa} / \%$ strain, $P=.04)$. At a higher frequency of $1.0 \mathrm{~Hz}$ and strain of $5 \%$, a significant reduction remained in the $\mathrm{STG}+\mathrm{EPC}$

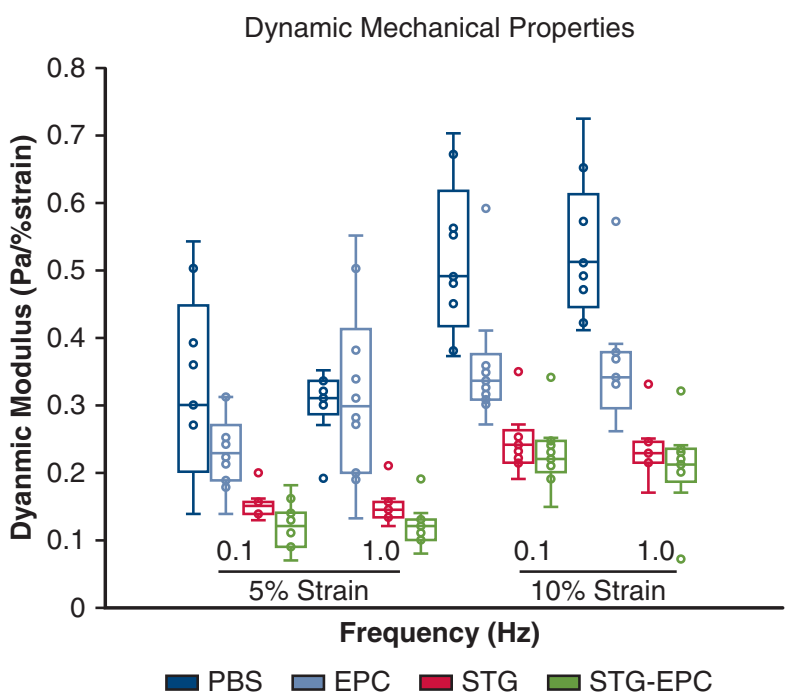

FIGURE 4. Dynamic mechanical properties of ventricular myocardial specimens from hearts injected with PBS $(n=9)$, EPCs $(n=10)$, STG $(\mathrm{n}=9)$, or STG-EPC $(\mathrm{n}=11)$. The dynamic modulus is the ratio of stress (Pa) to \% strain. The middle horizontal line represents the median for each group. The upper and lower whiskers represent the maximum and minimum values of nonoutliers. Extra dots data points represent outliers. $E P C$, Endothelial progenitor cell; $P B S$, phosphate-buffered saline; $S T G$, shear-thinning gel. group $(0.18 \pm 0.13 \mathrm{~Pa} / \%$ strain $)$ compared with $\mathrm{PBS}$ (0.45 $\pm 0.32 \mathrm{pa} / \%$ strain $),$ EPC $(0.36 \pm 0.33 \mathrm{pa} / \%$ strain $)$, and STG groups $(0.21 \pm 0.14 \mathrm{pa} / \%$ strain, $P=.03)$. At $10 \%$ strain and a frequency of $0.1 \mathrm{~Hz}$, a similar reduction in stress was observed within the STG + EPC group $(0.25 \pm 0.16 \mathrm{pa} / \%$ strain $)$ compared with PBS $(0.57 \pm 0.38 \mathrm{pa} / \%$ strain $)$, EPC $(0.43 \pm 0.35 \mathrm{pa} / \%$ strain $)$, and STG $(0.30 \pm 0.17 \mathrm{pa} / \%$ strain, $P=.04)$. At $1.0 \mathrm{~Hz}$ and $10 \%$ strain, less strain was observed in the STG + EPC group $(0.27 \pm 0.17 \mathrm{pa} / \%$ strain $)$ compared with PBS $(0.61 \pm 0.39 \mathrm{pa} / \%$ strain $)$, EPC $(0.47 \pm 0.37$ $\mathrm{pa} / \%$ strain $)$, and STG $(0.32 \pm 0.18 \mathrm{pa} / \%$ strain, $P=.3)$.

\section{Bioluminescent Tracking of Delivered Endothelial Progenitor Cells}

Retention of NIR-815-labeled EPC cells at the heart apex after injection of STG + EPC is shown in Figure 5 compared with isolated injection of labeled EPC cells. After 24 hours, retention of the cells was seen within the targeted myocardium. After 14 days, tagged cells remain within the myocardium. As noted, there is a dramatic reduction of cells within 24 hours after EPC-only injection at all observed time points.

\section{DISCUSSION}

Regional mechanical changes in the myocardium must be considered when designing a targeted biomaterial treatment for MI stabilization and functional improvement. Previous biomaterial studies designed to alter myocardial mechanics post-MI have demonstrated that injectable materials lead to thicker, stiffer infarcts, with limited infarct expansion and preserved LV geometry. ${ }^{25-28}$ As shown in the AUGMENT-HF trial, surgical treatment with Algisyl, an alginate-based polymer, was effective in improving exercise capacity and symptoms in patients with advanced HF, with an acceptable safety profile. ${ }^{29}$ It is clear the LV augmentation provides hemodynamic benefits.

Current treatments are focused on incorporation of both a cellular and biomaterial component. As a result, a better 


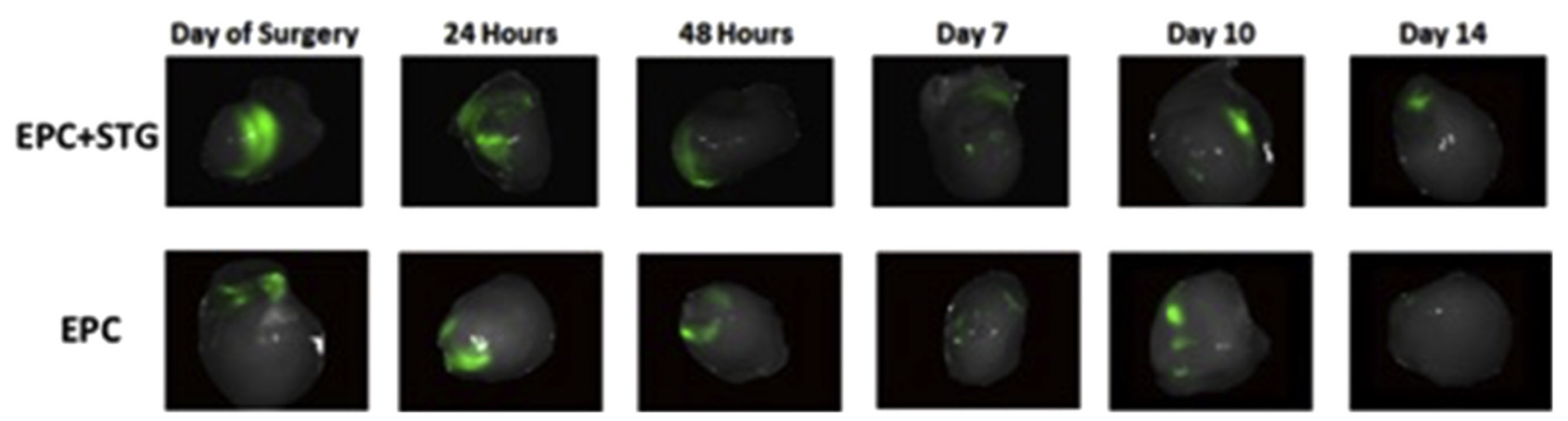

FIGURE 5. Fluorescently NIR 815 tagged EPCs apical view (border zone and ischemic myocardium) from day of surgery to 14 days after MI comparing $\mathrm{EPC}+\mathrm{STG}$ delivery with EPC alone delivery. EPC, Endothelial progenitor cell; STG, shear-thinning gel.

understanding between regional myocardial improvement, retention of cellular delivery, and improved global outcomes is needed to assess the effectiveness of the therapy design. Toward this goal, our work used speckle-tracking echocardiography, uniaxial testing, and therapeutic cell retention assessment to examine the impact of a combined cellular and hydrogel therapy on infarcted and border zone myocardium.

Localized ventricular restraint and injectable biomaterials have gained considerable interest to target and reduce adverse remodeling in heart failure. The interest stems from the premise that infarct expansion, which occurs as the heart dilates and myocardial wall thins, precipitates and sustains remodeling. ${ }^{3}$ The post-MI border zone represents a therapeutic target for inhibiting deleterious ventricular myocardial remodeling. As noted by Zhang and colleagues, ${ }^{30}$ improvement of the border zone function could lead to improvement in pump function. One of the primary goals of myocardial imaging is to identify dysfunctional, yet salvageable, myocardial segments that may return to normal function after revascularization. Strain echocardiography enables the study of myocardial function independently of the ultrasound beam direction. ${ }^{31}$ This provides quantification of regional myocardial systolic function with higher efficiency than Doppler tissue imaging ${ }^{32}$ and with higher specificity than conventional measurements. ${ }^{33-35}$

The major finding of our study was that myocardial injections of the STG + EPC construct reduced LV dilatation and was associated with increased border zone and global LV function compared with the isolated treatment with STG or EPC (Figure 6). Through the injection of the combination treatment of STG + EPC, it was observed that the ventricle did not dilate or thin suggesting that the infarct did not expand. Because of stabilization of the infarcted myocardium, global function was maintained in the STG + EPC treatment group as evidenced by the preservation of ejection fraction and contractility.
These findings were accompanied by normalization of myocardial velocity with a subsequent increase in the ability to withstand myocardial strain in the border zone compared with the remote myocardium when treated with the STG + EPC. Through velocity normalization, the dynamic myocardium continues to contribute the global cardiac function. In addition, as evidenced by the normalization of measurements, the STG + EPC-treated myocardium retained the lengthening properties to contribute to global function. Within the STG, improved strain measurements were also observed and are likely due to immediate infarct stabilization. The lack of improvement in other measurements within the STG cohort is likely due to the absence of neovascularization mediated through the EPCs as was observed in the $\mathrm{STG}+\mathrm{EPC}$ construct.

Assessment of regional stress demonstrates that the STG + EPC treatment injection leads to increased myocardial stiffness in the infarct region relative to controls. Immediately post-MI, the infarct loses its systolic contractile function, whereas diastolic function is relatively maintained. This abrupt transition from an active to a passive function leads to immediate stretching and thinning of the infarct, as well as LV diltation. ${ }^{36}$ The retention of myocardial strength observed with the STG and STG + EPC treatments indicates that the hydrogel alone is able to alter the biomechanical properties of the infarct before the body's natural compensation to ischemia; this effect is even more pronounced when the hydrogel and cellular components are combined. The contribution of the hydrogel is to reduced infarct expansion and LV dilation, likely through reduction in the load of individual muscle fibers. The normalization of the myocardial stiffness that is observed by gel injection alone is likely due to a biological response to the material. ${ }^{37}$ From the prior hydrogel characterization, this selected formulation of the STG degrades before 4 weeks; yet the geometry and mechanical properties of the myocardium are maintained in the STG and 


\section{Delivery of Endothelial Progenitor Cells (EPCs) with Injectable Shear-Thinning Gel (STG) in Myocardial Ischemia}

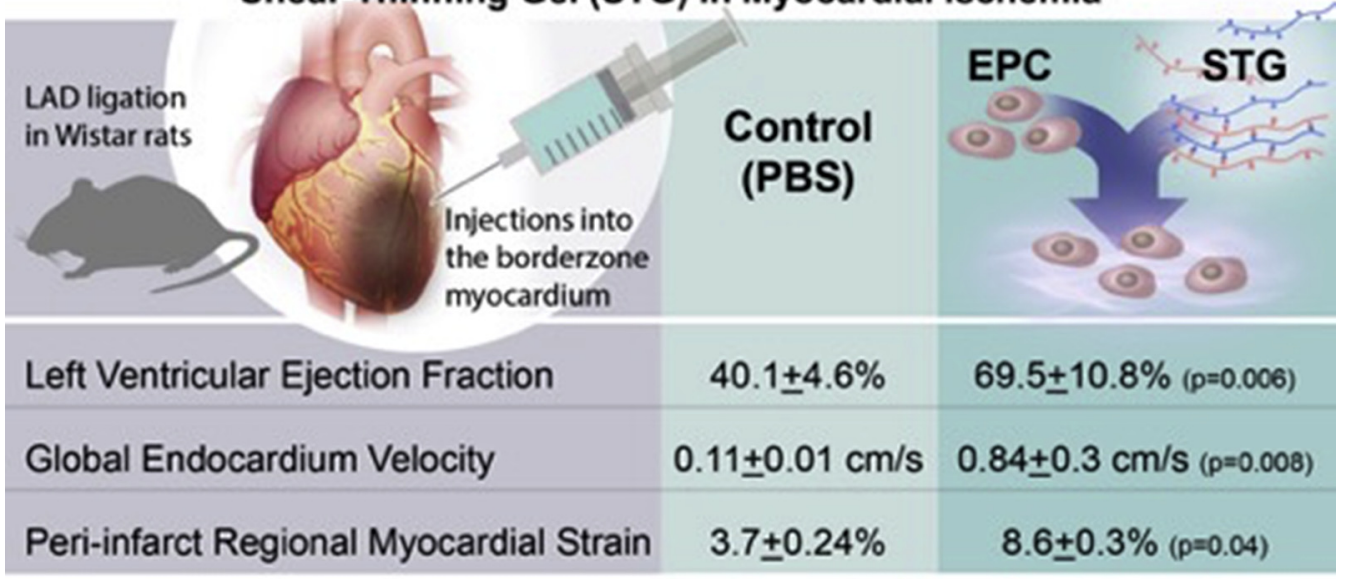

Gaffey AC et al. The Journal of Thoracic and Cardiovascular Surgery 2018

FIGURE 6. Schematic displaying the injection of the STG + EPC construct into the ligated LAD of the Wistar rats. Results note a significant improvement with construct treatment of STG + EPC compared with PBS alone as noted by the improvement in LV ejection fraction, global endocardium velocity, and peri-infarct regional myocardial strain. EPC, Endothelial progenitor cell; $P B S$, phosphate-buffered saline; $S T G$, shear-thinning gel; $L A D$, left anterior descending.

STG + EPC compared with the controls. ${ }^{6}$ This maintenance of the myocardial function suggests a response to the STG gel, which includes the degradation products of the HAbased gel or an inflammatory response that leads to enhanced cellular proliferation.

In this report, we describe the utility of noninvasive imaging methods for highly sensitive and rapid assessment of myocardial biomechanics. The advanced speckletracking-based strain measures of LV performance are not only more sensitive than conventional echocardiographic measures in detecting global changes in post-MI cardiac function, ${ }^{35}$ but also allow for region-specific functional assessment in infarcted, border zone, and noninfarcted areas of the myocardium. Normal LV function involves a typical pattern of myocardial deformation that includes longitudinal shortening, circumferential shortening, and radial thickening in systole, followed by reverse changes in diastole. ${ }^{38,39}$ Compared with strain measurements in the circumferential or radial axes, which predominately reflect activity of the mesocardium (mid-myocardium), longitudinal strain is a particularly sensitive marker of the subendocardial myofiber dysfunction. It is such subendocardial myofiber dysfunction that tends to occur early in the setting of hypoperfusion or mechanical stress. ${ }^{40-42}$ Accordingly, we observed that regional and global measures of longitudinal strain significantly decreased after treatment with STG + EPC.
We further quantified the changes in the viscoelastic material properties of cardiac muscle that occurred as a result of MI and the modification of these properties after treatment with EPCs and shear-thinning hydrogel. The STG alters the mechanics of the border zone myocardium. The samples tested were taken from longitudinally oriented ventricular muscle biopsies as opposed to circumferentially oriented specimens to minimize the effects of the natural curvature of the sample in the circumferential direction. We have shown that our STG + EPC treatment limits expansion of the infarct by neovascularization of the border zone. This increase in angiogenesis shown in our prior work ${ }^{6}$ preserves the extracellular matrix and ultimately confers mechanical behaviors on the treated muscle that are more similar to the native tissue. A reduction in tensile modulus of the tissue correlates to a decrease in LV wall stress that prevents the progression toward adverse ventricular remodeling.

Overall, the normalization in global function in the STG + EPC cohort can be due to the targeted delivery and retention of the EPCs at the vulnerable border zone myocardium. As seen in the near-infrared imaging, there is a dramatic loss of the EPCs within 24 hours when injected as an isolated component. Through seeding of the STG with EPCs, retention of the cells within the myocardium is observed at 14 days. It is through this retention within the myocardium that the EPCs are able to exert a greater effect 
on the vulnerable myocardium and preserve the systolic function for the at-risk myocardium.

\section{Study Limitations}

Several limitations of this study merit consideration. Sources of variation exist in speckle-tracking-based strain analysis, including the echocardiographic views obtained for image analysis. Changes in the imaging angle of incidence can result in the capturing of different myocardial sarcomere fibers at different levels, and thus yield variable results. However, because of the presence of multiple orientation points in the long-axis views including the LV outflow tract, mitral valve leaflet plane, and LV apex, this view is less prone to angular variation. Another source of variability is the tracing performed by the investigator, because strain values are dependent on the quality and location of the tracing. Through the use of 2 independent investigators and ensuring minimal interobserver variation, any significant variation would be accounted, and measurements differing by greater than $10 \%$ would be repeated. In addition, there is a risk of a type I error within the study of $5 \%$ risking that the null hypothesis was rejected.

\section{CONCLUSIONS}

The novel tissue-engineered STG used in this study may improve therapies by allowing investigators to stabilize the at-risk border zone myocardium, to maintain ventricular geometry, to enhance the delivery of cellular therapy with significant cellular retention at targeted sites, and to improve ventricular hemodynamics. We have shown improvements in global ventricular function, regional border zone strain, and retention of ventricular velocity after injection of the STG + EPC into the border zone of ischemic myocardium.

We believe that several avenues of research could be explored as a next step in this therapeutic strategy, including noninvasive cell tracking, definition of paracrine mechanisms, and percutaneous delivery. We believe that there will be a transition away from cellular delivery to that of extracellular vesicles as future studies for the advancement of clinical translatability. Through the use of extracellular vesicles, the obstacles associated with cellular deliver will be eliminated, and perhaps the paracrine factors will provide the same improvements as direct cellular delivery. Furthermore, before clinical translation, preclinical large animal trials are warranted. Clinically, the STG is an exciting method to permit targeted delivery of cell-based treatment. We present the use of STG as a delivery vehicle for EPC-mediated therapy to provide gelmediated myocardial stabilization. Through this study, we have shown the following: Biocompatible and injectable STG can improve LV hemodynamics, maintain ventricular geometry, limit adverse remodeling, and improve cardiac function.

\section{Webcast}

You can watch a Webcast of this AATS meeting presentation by going to: https://aats.blob.core.windows.net/media/ 17AM/2017-05-01/Booth-106/05-01-17_CTTheaterI_Booth106_1305_Gaffey.mp4.

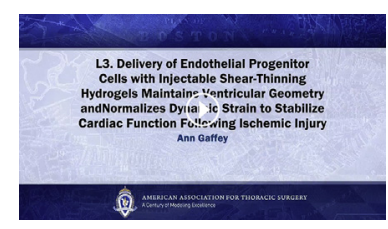

\section{Conflict of Interest Statement}

Authors have nothing to disclose with regard to commercial support.

\section{References}

1. Mozaffarian D, Benajmin A, Gao A, Arnett D, Blaha J, Cushman M, et al Heart disease and stroke statistics-2015 update: a report from the American Heart Association. Circulation. 2014;131:e29-322.

2. Jackson BM, Gorman J, Salgo I, Moainie S, Plappert T, St John-Sutton M, et al. Border zone geometry increases wall stress after myocardial infarction: contrast echocardiographic assessment. Am J Physiol Heart Circ Physiol. 2003;284: H475-9.

3. Jackson BM, Gorman J, Moainine S, Guy T, Naru N, Narula J, et al. Extension of borderzone myocardium in postinfarction dilated cardiomyopathy. J Am Coll Cardiol. 2002;40:1160-7; discussion 1168-71.

4. Wenk JF, Eslami P, Zhang Z, Xu C, Kuhl E, Gorman J, et al. A novel method for quantifying the in-vivo mechanical effect of material injected into a myocardial infarction. Ann Thorac Surg. 2011;92:935-41.

5. Yu J, Christman KL, Chin E, Sievers RE, Saeed M, Lee RJ. Restoration of left ventricular geometry and improvement of left ventricular function in a rodent model of chronic ischemic cardiomyopathy. J Thorac Cardiovasc Surg. 2009; 137:180-7.

6. Gaffey AC, Chen M, Venkataraman C, Trubelja A, Rodell C, Dinh P, et al. Injectable shear-thinning hydrogels used to deliver endothelial progenitor cells, enhance cell engraftment, and improve ischemic myocardium. J Thorac Cardiovasc Surg. 2015;150:1268-77.

7. Leone AM, tella S, Bonanno G, Abbate A, Rebuzzi A, Giovannini S, et al. Mobilization of bone marrow-derived stem cells after myocardial infarction and left ventricular function. Eur Heart J. 2005;26:1196-204.

8. Atluri P, Trubelja A, Fairman A, Hsiao P, Macarthur J, Cohen J, et al. Normalization of postinfarct biomechanics using a novel tissue-engineered angiogenic construct. Circulation. 2013;128(11 Suppl 1):S95-104.

9. Dawn B, Bolli R. Adult bone marrow-derived cells: regenerative potential, plasticity, and tissue commitment. Basic Res Cardiol. 2005;100:494-503.

10. Abdel-Latif A, Bolli R, Tleyjeh I, Montori V, Perin E, Hornung C, et al. Adult bone marrow-derived cells for cardiac repair: a systematic review and meta-analysis. Arch Intern Med. 2007;167:989-97.

11. Hofmann M, ollert K, Meyer G, Menke A, Arseniev L, Hertenstein B, et al Monitoring of bone marrow cell homing into the infarcted human myocardium. Circulation. 2005;111:2198-202.

12. Sheikh AY, Huber B, Narsinh K, Spin J, Van Der Bogt K, De Almeida P, et al. In vivo functional and transcriptional profiling of bone marrow stem cells after transplantation into ischemic myocardium. Arterioscler Thromb Vasc Biol. 2012;32:92-102.

13. Dash R, Kim P, Matsuura Y, Ikeno F, Metzler S, Huang N, et al. Manganese-enhanced magnetic resonance imaging enables in vivo confirmation of peri-infarct restoration following stem cell therapy in a porcine ischemia-reperfusion model. J Am Heart Assoc. 2015;4.

14. Blocki A, Beyer S, Dewavrin J, Goralczyk A, Wang Y, Peh P, et al. Microcapsules engineered to support mesenchymal stem cell (MSC) survival and proliferation enable long-term retention of MSCs in infarcted myocardium. Biomaterials. 2015;53:12-24. 
15. Freeman BT, Kouris NA, Ogle BM. Tracking fusion of human mesenchymal stem cells after transplantation to the heart. Stem Cells Transl Med. 2015;4:685-94.

16. Rodell CB, Kaminski AL, Burdick JA. Rational design of network properties in guest-host assembled and shear-thinning hyaluronic acid hydrogels. Biomacromolecules. 2013;14:4125-34.

17. Frederick JR, Fitzpatrick J, McCormick R, Harris D, Kim A, Muenzer J, et al. Stromal cell-derived factor-1alpha activation of tissue-engineered endothelial progenitor cell matrix enhances ventricular function after myocardial infarction by inducing neovasculogenesis. Circulation. 2010;122:S107-17.

18. Atluri P, Miller J, Emery R, Hung G, Trubelja A, Cohen J, et al. Tissue-engineered, hydrogel-based endothelial progenitor cell therapy robustly revascularizes ischemic myocardium and preserves ventricular function. J Thorac Cardiovasc Surg. 2014;148:1090-7; discussion 1097-8.

19. Liu YH, Yang X, Nass O, Sabbah H, Peterson E, Carretero O, et al. Chronic heart failure induced by coronary artery ligation in Lewis inbred rats. Am J Physiol. 1997;272:H722-7.

20. Woo YJ, Panlilio C, Cheng R, Liao G, Atluri P, Hsu V, et al. Therapeutic delivery of cyclin A2 induces myocardial regeneration and enhances cardiac function in ischemic heart failure. Circulation. 2006;114:I206-13.

21. MacArthur JW, Trubelja A, Shudo Y, Hsiao P, Fairman A, Yang E, et al. Mathematically engineered stromal cell-derived factor- $1 \alpha$ stem cell cytokine analog enhances mechanical properties of infarcted myocardium. J Thorac Cardiovasc Surg. 2013;145:278-84.

22. Miller EJ, Kent Rhodes R. Structural and contractile proteins part A: extracellular matrix. In: Methods in Enzymology, Volume 82. Philadelphia, PA: Elsevier; 1982.

23. Bermudez DM, Herdrich B, Xu J, Lind R, Beason D, Mitchell M, et al. Impaired biomechanical properties of diabetic skin implications in pathogenesis of diabetic wound complications. Am J Pathol. 2011;178:2215-23.

24. Dunkman AA, Buckley M, Mienaltowski M, Adams S, Thomas S, Kumar A, et al. The injury response of aged tendons in the absence of biglycan and decorin. Matrix Biol. 2014;35:232-8.

25. Ifkovits JL, Tous E, Minakawa M, Morita M, Robb D, Koomalsingh K, et al. Injectable hydrogel properties influence infarct expansion and extent of postinfarction left ventricular remodeling in an ovine model. Proc Natl Acad Sci. 2010;107:11507-12

26. Highley CB, Prestwich GD, Burdick JA. Recent advances in hyaluronic acid hydrogels for biomedical applications. Curr Opin Biotechnol. 2016; 40:35-40.

27. Mukherjee R, Zavadzkas J, Saunders S, McLean J, Jeffords L, Beck C, et al. Targeted myocardial microinjections of a biocomposite material reduces infarct expansion in pigs. Ann Thorac Surg. 2008;86:1268-76.

28. Landa N, Miller L, Feinberg M, Holbova R, Shachar M, Freeman I, et al. Effect of injectable alginate implant on cardiac remodeling and function after recent and old infarcts in rat. Circulation. 2008;117:1388-96.

29. Anker SD, Coats A, Cristian G, Dragomir D, Pusineri E, Piredda M, et al. A prospective comparison of alginate-hydrogel with standard medical therapy to determine impact on functional capacity and clinical outcomes in patients with advanced heart failure (AUGMENT-HF trial). Eur Heart J. 2015;36: 2297-309.

30. Zhang Z, Sun K, Saloner D, Wallace A, Ge L, Baker A, et al. The benefit of enhanced contractility in the infarct borderzone: a virtual experiment. Front Physiol. 2012;3:86.

31. Motoki H, Nakatani S, Abe H, Kanzaki H, Kitakaze M. Heterogeneous contraction of the left ventricle demonstrated by 2-dimensional strain imaging. J Echocardiogr. 2009;8:33-9.

32. Sahlen A, Winter R. How should we measure global and regional left ventricular systolic function? J Echocardiogr. 2011;9:41-50.

33. Piegari E, Di Salvo G, Castaldi B, Vitelli M, Rodolico G, Golino P, et al. Myocardial strain analysis in a doxorubicin-induced cardiomyopathy model. Ultrasound Med Biol. 2008;34:370-8.

34. Migrino RQ, Aggarwal D, Konorev E, Brahmbhatt T, Bright M, Kalyanaraman B. Early detection of doxorubicin cardiomyopathy using twodimensional strain echocardiography. Ultrasound Med Biol. 2008;34:208-14.

35. Bauer M, Cheng S, Jain M, Ngoy S, Theodoropoulos C, Trujillo A, et al. Echocardiographic speckle-tracking based strain imaging for rapid cardiovascular phenotyping in mice. Circ Res. 2011;108:908-16.

36. Holmes JW, Borg TK, Covell JW. Structure and mechanics of healing myocardial infarcts. Annu Rev Biomed Eng. 2005;7:223-53.

37. Anderson JM, Rodriguez A, Chang DT. Foreign body reaction to biomaterials. Semin Immunol. 2008:20:86-100.
38. Sengupta PP, Krishnamoorthy V, Korinek J, Narula J, Vannan M, Lester S, et al. Left ventricular form and function revisited: applied translational science to cardiovascular ultrasound imaging. J Am Soc Echocardiogr. 2007;20:539-51.

39. Rosen BD, Gerber B, Edvardsen T, Castillo E, Amado L, Nasir K, et al. Late systolic onset of regional LV relaxation demonstrated in three-dimensional space by MRI tissue tagging. Am J Physiol Heart Circ Physiol. 2004;287:H1740-6.

40. Cottrell C, Kirkpatrick JN. Echocardiographic strain imaging and its use in the clinical setting. Expert Rev Cardiovasc Ther. 2010;8:93-102.

41. Sabbah HN, Marzilli M, Stein PD. The relative role of subendocardium and subepicardium in left ventricular mechanics. Am J Physiol. 1981;240:H920-6.

42. Hashimoto I, Li X, Hejmadi Bhat A, Jones M, Zetts A, Sahn D, et al. Myocardial strain rate is a superior method for evaluation of left ventricular subendocardial function compared with tissue Doppler imaging. J Am Coll Cardiol. 2003;42: 1574-83.

Key Words: cellular therapy, hydrogel, ischemic myocardium, shear-thinning gel

\section{Discussion}

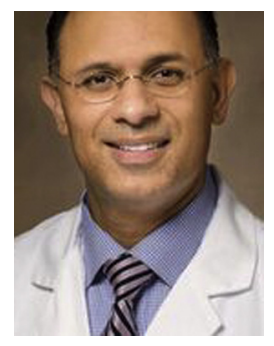

Dr Khalpey, MD (Tucson, Ariz). Did you see any increase in muscle mass and if you did, could you have done a magnetic resonance imaging (MRI) scan, because speckle tracking takes forever, and I would tear my hair out. So why didn't you use MRI?

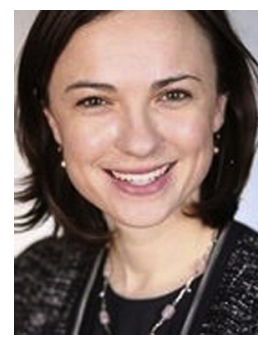

Dr Gaffey. I think MRI is great but time-consuming. So for us, the speckle tracking was easy to obtain. We had 2 independent users assessing it. We were easily able to scan through 40 animals in a matter of 2 days to assess it. But I think MRI would only further supplement additional strain modulus compared with the speckle tracking.

Dr Khalpey. Would it have given you a bit more architecture about what was going on and if you truly injected in the border zone?

Dr Gaffey. Yes. In our initial study that was published in the Journal when we looked at ventricular geometry and dilatation, we were able to see that when we did histology at that point.

Dr Khalpey. So, $40 \%$ of endothelial cells at the time of an infarct migrate to become fibroblasts. That's a mechanism called epithelial mesenchymal transition. So when you injected the antigen-presenting cells, did you think about what these cells may become over 4 weeks, and if so, what would you have thought they would have become and how would the architecture by the gel help you in preserving and confirming some of the hypotheses that you found?

Dr Gaffey. One of the benefits of the gel, as you have mentioned, is [inaudible].

Dr Khalpey. This has strong clinical relevance, and what would your next preclinical model or your translatable model into a clinical trial be? 
Dr Gaffey. Currently we are about to start with a sheep model, and our sheep model will be an open, also a thoracotomy, with LAD ligation, but the benefit of this STG, and one of the things I didn't show, is that you are able to deliver it over a long distance. Ultimately, we could go percutaneously at the time of placement of a percutaneous coronary intervention for injection and to deliver our cells.

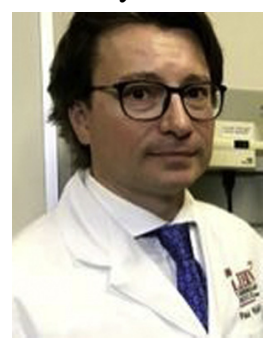

Dr P. Fedak (Calgary, Alberta, Can$a d a)$. The big question I have for you is whether you think these results are due to a biomechanical effect or a bioactive effect? For example, with cell therapy we usually see a response due to paracrine factors. I don't see that delivery measured in the myocardium. Have you measured those and is it possible that it is a combination of both, that you have got a change in the biomechanical effect plus maybe the cells are interacting with the gel in some way and becoming more paracrine?

Dr Gaffey. Exactly. The HA in and of itself is angiogenic. Once the STG is injected into the myocardium, it provides some stabilization of the border zone. I think the next step is to look at paracrine effects. It is probably a combination of cellular delivery, but there is a greater barrier to isolating the cells, but it is a paracrine-mediated effect, which our lab is currently looking at and one of my colleagues has presented at the American Heart Association with good results. 